\title{
Antimicrobial, Antioxidant and Antibiofilm Potential of Peppermint (Mentha piperita) Essential Oil for Application in Meat Products
}

\author{
Prabhjot Kaur ${ }^{1}$, Nitin Mehta ${ }^{1 *}$, Om Prakash Malav ${ }^{1}$, Manish Kumar Chatli ${ }^{1}$ and Harsh Panwar ${ }^{2}$ \\ ${ }^{1}$ Department of Livestock Products Technology, College of Veterinary Science, Guru Angad Dev Veterinary Animal Sciences \\ University, Ludhiana, Punjab, INDIA \\ ${ }^{2}$ Department of Dairy Microbiology, College of Dairy Science and Technology, Guru Angad Dev Veterinary Animal Sciences \\ University, Ludhiana, Punjab, INDIA \\ *Corresponding author: N Mehta; E-mail: nmvets220@gmail.com
}

Received: 31 Dec., 2019

Revised: 18 Jan., 2020

Accepted: 22 Jan., 2020

\begin{abstract}
The current study was carried out to examine in-vitro antimicrobial, antioxidant and antibiofilm efficacy of Peppermint essential oil (PEO) for its potential application in meat products. Antimicrobial activity was measured by using zone inhibition assay (ZOI) and Minimum Inhibitory Concentration (MIC) against ten food borne pathogens including four Gram-positive viz. Listeria monocytogenes, Enterococcus faecalis, Bacillus cereus and Staphylococcus aureus and six Gram-negative viz. Proteus mirabilis, Salmonella enterica serovar Typhi, Klebsiella pneumonia, Shigella flexneri, Escherichia coli and Pseudomonas aeruginosa whereas antioxidant assay was measured using 1,1 diphenyl-2 picrylhydrazyl (DPPH) and 2-2- azinobis-3 ethylbenthiazoline-6-sulphonic acid (ABTS) radical scavenging activity. Maximum zone size was observed for Proteus mirabilis whereas, MIC values ranged from 5000-20000 ppm for all tested organisms. The oil was found to be more effective against Pseudomonas aeruginosa and Salmonella enterica serovar Typhi. Antibiofilm activity (\%) was also performed against pure cultures of two pathogens i.e. Listeria monocytogenes and Proteus mirabilis as positive control. The results exhibited that with application of PEO, biofilm formation of both Listeria monocytogenes and Proteus mirabilis was inhibited by $45.80 \%$ and $73.01 \%$, respectively in contrast to their respective controls. ABTS and DPPH radical scavenging activity of PEO was measured at five different concentrations and values were ranging from $17.24-49.07 \%$ for ABTS whereas $35.16-60.70 \%$ for DPPH under investigation. It can be concluded that peppermint essential oil possesses potent antimicrobial, antioxidant and antibiofilm activity and can be further used as a natural alternative for preservation in meat industry.
\end{abstract}

Keywords: Peppermint essential oil, ZOI, MIC, DPPH, ABTS, antibiofilm activity, meat

An ideal source of quality animal protein of high biological value and other essential nutrients, meat has bagged a key position in human diet (Mehta et al., 2013). But it is an ideal substrate for the growth of food borne pathogens, therefore it is more prone to bacterial spoilage and enhances safety concerns amongst consumers. It is mandatory to ensure its safety prior to its consumption. Utilization of antimicrobials and antioxidants in meat matrix for extension of shelf life and control of microbial degradation is considered as a remedial measure (Verma et al., 2018). Although various synthetic antioxidants such as butylated hydroxyanisole (BHA), butylated hydroxytoluene (BHT), tert-butylhydroquinone (TBHQ), and propyl gallate (PG) have already been used as in the meat products (Jayathilakan et al., 2007), but they have many potential toxicological side-effects on consumer's health (Raghavan et al., 2007). Therefore, there has been a growing interest in the exploration of new effective antioxidants and antimicrobial compounds which have their origin from natural sources. In response to recent demand for natural products and increasing purchasing power of consumers for natural foods (Sebranek et al.,

\footnotetext{
How to cite this article: Kaur, P., Mehta, N., Malav, O.P., Chatli, M.K. and Panwar, H. (2020). Antimicrobial, antioxidant and antibiofilm potentia of peppermint (Mentha piperita) essential oil for application in meat products. J. Anim. Res., 10(1): 33-40.
} 
2007), the meat industry is trying actively to search for various natural methods in order to control oxidative rancidity and enhancement of the shelf life of meat products (Naveena et al., 2008). In order to solve this problem, various phytoextracts, phytochemicals and essential oils obtained from plants that are considered to be safe for human health are increasingly being tried in food industries.

Essential oils (EOs) are known as the complex mixtures of low molecular weight compounds which are extracted from diverse plants by using steam distillation and various other solvents. The biological properties and distinctive aroma of essential oils are due to its major components i.e. terpenoids and phenylpropanoids (Raut et al., 2014). They are used in a various fields such as food, pharmaceutical and cosmetics industries due to their flavoring, antimicrobial and antioxidant properties (Adorjan et al., 2010). The phenolic compounds of essential oils attributes to their antimicrobial action as they enter the microbial cell membrane and causes leakage of ions and cytoplasmatic content which leads to the cellular breakdown (Burt, 2004). As essential oils are recognized as safe natural compounds and potential alternative to synthetic additives therefore they can be used in foods for preservation (Shree et al., 2019).

Peppermint essential oil (PEO) is derived from the leaves of a perennial herb i.e. Mentha piperita which belongs to family Lamiaceae and grows throughout Europe and North America. It is reported to have several valuable attributes such as antiviral, antimicrobial, antioxidant, slightly anaesthetic, anti-inflammatory, antispasmodic, antiulcer and hepatoprotective (Shah et al., 2004). The major content in peppermeint essential oil is menthol which constitutes $30-55 \%$ of the total compounds of the oil which mainly contributes to its antioxidant and antimicrobial actions in various food systems (Kamatou et al., 2013; Kumar et al., 2011; Kanatt et al., 2008) and it have also been observed to be moderately effective against both Gram-negative and Gram-positive food borne pathogens (Saeed et al., 2006). Though it has numerous medicinal properties and is considered to be safe, still very limited work has been carried out with respect to its application in meat industry. Keeping all points in view, the aim of this study was to investigate and evaluate the in-vitro antimicrobial, antioxidant and antibiofilm activity of peppermint essential oil for their possible application in meat products.

\section{MATERIALS AND METHODS}

\section{Source of Peppermint Essential oil}

Peppermint oil was procured from Kanta Enterprises Pvt. Ltd, Noida, UP, India. As per the certificate of analysis provided with the product, it has refractive index in range of 1.449-1.467 and has specific gravity in range of 0.8900.905 . The oil contained $39.50 \%$ of menthol as its main constituent. All the reagents and chemicals used in the study were of analytical grade.

\section{Bacterial strains and growth conditions}

Ten pure freeze dried cultures were procured from Institute of Microbial Technology (IMTECH), Chandigarh, India viz. Bacillus cereus (MTCC 1272), Escherichia coli (MTCC 723), Enterococcus faecalis (MTCC 890), Klebsiella pneumoniae (MTCC 109), Listeria monocytogenes (MTCC 1143), Proteus mirabilis (MTCC 425), Pseudomonas aeruginosa (MTCC 741), Salmonella enterica serovar Typhi (MTCC 733), Shigella flexneri (MTCC 1457), and Staphylococcus aureus (MTCC 96). These cultures were revived and stock cultures were prepared and are being maintained at $-80^{\circ} \mathrm{C}$ in the department by regular passaging.

\section{Antimicrobial activity estimation}

Antimicrobial potential of Peppermint essential oil (PEO) was tested on ten entero-pathogenic bacterial cultures (Bacillus cereus, Escherichia coli, Enterococcus faecalis, Klebsiella pneumonia, Listeria monocytogenes, Proteus mirabilis, Pseudomonas aeruginosa, Salmonella enterica, Shigella flexneri and Staphylococcus aureus), which were cultured into fresh broth media and brought into log phase of growth by incubating at $37^{\circ} \mathrm{C}$ for 24 hours before being used. The antibacterial activity was assessed using Disc diffusion assay (Bauer et al., 1966). Mueller-Hinton agar (HiMedia Laboratories) plates were inoculated with one of the selected bacterial species using a sterile swab dipped into a bacterial suspension containing $10^{7} \mathrm{CFU} /$ $\mathrm{ml}$. Then a sterile paper disk (5 mm ID, Whatmann \#1) 
was placed onto the centre of the agar plate and $5 \mu \mathrm{l}$ of EO was placed onto the centre of the disk. Plates were then incubated at $37^{\circ} \mathrm{C}$ for $24 \mathrm{~h}$. Following incubation, growth inhibition zones were observed. Diameters of these zones were measured and tabulated.

\section{Minimum Inhibitory Concentration (MIC)}

It was estimated by method followed by Kumar et al. (2017) with some modifications. Uniform concentrations of log phase bacterial cultures were prepared by adjusting their absorbance at $600 \mathrm{~nm}$. Concentrations of Peppermint essential oil were adjusted to $0.125 \%, 0.25 \%, 0.1 \%, 0.3 \%$, $0.5 \%, 1.0 \%, 2.0 \%$ and $3.0 \%$ with help of DMSO. In the Microtiter plates, $100 \mu \mathrm{l}$ of each culture were added to 30 $\mu 1$ of oil dilution and $170 \mu \mathrm{l}$ of nutrient broth. The plates were kept for incubation at $37^{\circ} \mathrm{C}$ for 24 hours. Absorbance of samples was measured at $600 \mathrm{~nm}$ to observe growth inhibition. The growth inhibition was also confirmed by streaking the samples on nutrient agar plates and observing for bacterial growth after 24 hours incubation at $37^{\circ} \mathrm{C}$.

\section{Antioxidant Activity Assay}

The antioxidant activity of the peppermint essential oil was measured as free radical scavenging activity. The ability of essential oil to donate hydrogen atoms or electrons was evaluated from bleaching of coloured methanoloic solution of 1,1-diphenyl-2-picrylhydrazyl (DPPH) radical scavenging activity. The possibility of free radical scavenging activity is higher if number of hydroxyl groups are more.

\section{1,1-diphenyl-2-picrylhydrazyl (DPPH) radical scavenging activity}

Radical scavenging potential of Peppermint Oil was assessed using a methanolic solution of the "stable" free radical, DPPH. The method of (Blois, 1958) was used in studying the effect of various oil concentrations on DPPH radicals with some modifications. A solution of DPPH $(0.15 \mathrm{mmol} / \mathrm{L})$ in methanol was prepared. Oil concentrations $(0.5,1.0,1.5,2.0$, and $2.5 \%)$ were prepared in methanol and $200 \mu \mathrm{l}$ of each dilution was mixed with $50 \mu 1$ of DPPH solution in a 96 well microtitre plates. The mixture was allowed to stand at room temperature in dark for $30 \mathrm{~min}$. The decrease in absorbance at $517 \mathrm{~nm}$ was measured. Butylated hydroxyanisole (BHA) was used as positive control. The radical scavenging activity was measured as a decrease in absorbance of DPPH.

$\%$ Radical Inhibition $=\{($ Control OD - Sample OD $) /$ Control OD $\} \times 100$

\section{2,2'-azinobis-3-ethylbenzthiazoline-6-sulphonate (ABTS) radical scavenging activity}

ABTS cation decolorization assay was conducted on various concentrations of Peppermint oil $(0.5,1.0,1.5$, 2.0, 2.5\%) made in methanol (Re et al., 1999) with slight modifications. ABTS radical cation was freshly prepared by mixing $14 \mathrm{mM}$ ABTS with equal volume of $4.95 \mathrm{mM}$ potassium persulphate and kept for 24 hours at room temperature. The ABTS radical cation was used for the assay after dilution with Phosphate Buffer Saline (PBS) appropriately. To $50 \mu \mathrm{l}$ of various concentrations of PEO, $150 \mu \mathrm{l}$ of ABTS solution was added. After $1 \mathrm{~min}$ incubation at room temperature, absorbance was measured at $732 \mathrm{~nm}$. Methanol was used as blank solution and ABTS solution without essential oil served as control. The cation scavenging activity was measured same as with DPPH.

$\%$ Radical Inhibition $=\{($ Control OD - Sample OD $) /$ Control OD $\} \times 100$

\section{Antibiofilm Activity}

Biofilm inhibition was achieved by aliquoting $10 \mu \mathrm{L}$ of a standardized $\left(\sim 10^{6} \mathrm{CFU} / \mathrm{ml}\right)$ L. monocytogenes and P.mirabilis culture into each $96-$ well microtitre plate followed by the addition of $30 \mu \mathrm{L}$ of PEO (1250 ppm), respectively (Galvao et al., 2012). Following incubation at $37{ }^{\circ} \mathrm{C}$ for $24 \mathrm{~h}$, the plates were rinsed three times with phosphate-buffered saline (PBS, $\mathrm{pH}$ 7.2) to remove loosely attached cells. The plates were air-dried and then the wells were stained with $250 \mu \mathrm{L}$ of $0.1 \%$ crystal violet and incubated at room temperature for $30 \mathrm{~min}$. After incubation, the plates were washed and then left to dry. Finally, $250 \mu \mathrm{L}$ of $33 \%$ glacial acetic acid was added to solubilize the dye and the $\mathrm{OD}_{570}$ was recorded using microplate reader. The per cent biofilm inhibition was estimated following the formula:

Anti-biofilm activity $(\%)=\frac{\text { Control } \mathrm{OD}_{570 \mathrm{~nm}}-\text { Test } \mathrm{OD}_{570 \mathrm{~nm}}}{{\text { Control } \mathrm{OD}_{570 \mathrm{~nm}}}_{100}} \times 100$ 
The positive control was the amount of biofilm formed with a pure culture of L. monocytogenes and P.mirabilis.

\section{Visualization of biofilms}

L. monocytogenes and P.mirabilis biofilms, with or without treatment with PEO, were grown on pre-sterilized glass cover slips (Musthafa et al., 2010). An aliquot of $80 \mu \mathrm{L} /$ well $\left(\sim 10^{6} \mathrm{CFU} / \mathrm{mL}\right)$ of overnight grown test pathogens were added to 6 well tissue culture plate containing sterile glass cover slips, BHI medium $(1680 \mu \mathrm{L} /$ well $)$ and PEO (240 $\mu \mathrm{L} /$ well). The bacterial suspensions were used as controls. Biofilms were stained with crystal violet and the stained biofilms were visualized under light microscope at 40X magnification (Olympus Microscope, USA).

\section{Statistical analysis}

Data was analyzed statistically on 'SPSS-16.0' (SPSS Inc., Chicago, II USA) software package as per standard methods (Snedecor and Cochran, 1994). Whole set of experiment was repeated three times and mean values were reported along with standard error. The statistical significance was estimated at 5\% level $(\mathrm{p}<0.05)$ and evaluated with Duncan's Multiple Range Test (DMRT).

\section{RESULTS AND DISCUSSION}

\section{Antimicrobial activity and MIC of Peppermint essential oil}

Antimicrobial activity was performed by disc diffusion method against ten foodborne pathogens (Fig. 1). Clear demarcated zone appeared which is an indicative of inhibition of various microorganisms by the peppermint oil used in our study.

Inhibition zone size of PEO ranged between 12.33 to $34.66 \mathrm{~mm}$ and maximum inhibition zone diameter was observed for Proteus mirabilis $(34.66 \pm 1.20)$ followed by Staphylococcus aureus $(26.66 \pm 1.76)$ whereas minimum inhibition zone diameters were observed for Klebsiella pneumonia $(12.33 \pm 0.88)$ followed by Salmonella enterica (14.33 \pm 1.20$)$ (Fig. 2). The MIC (ppm) of the oil against the above mentioned organisms is presented in Fig. 3. MIC (ppm) of peppermint essential oil against the tested

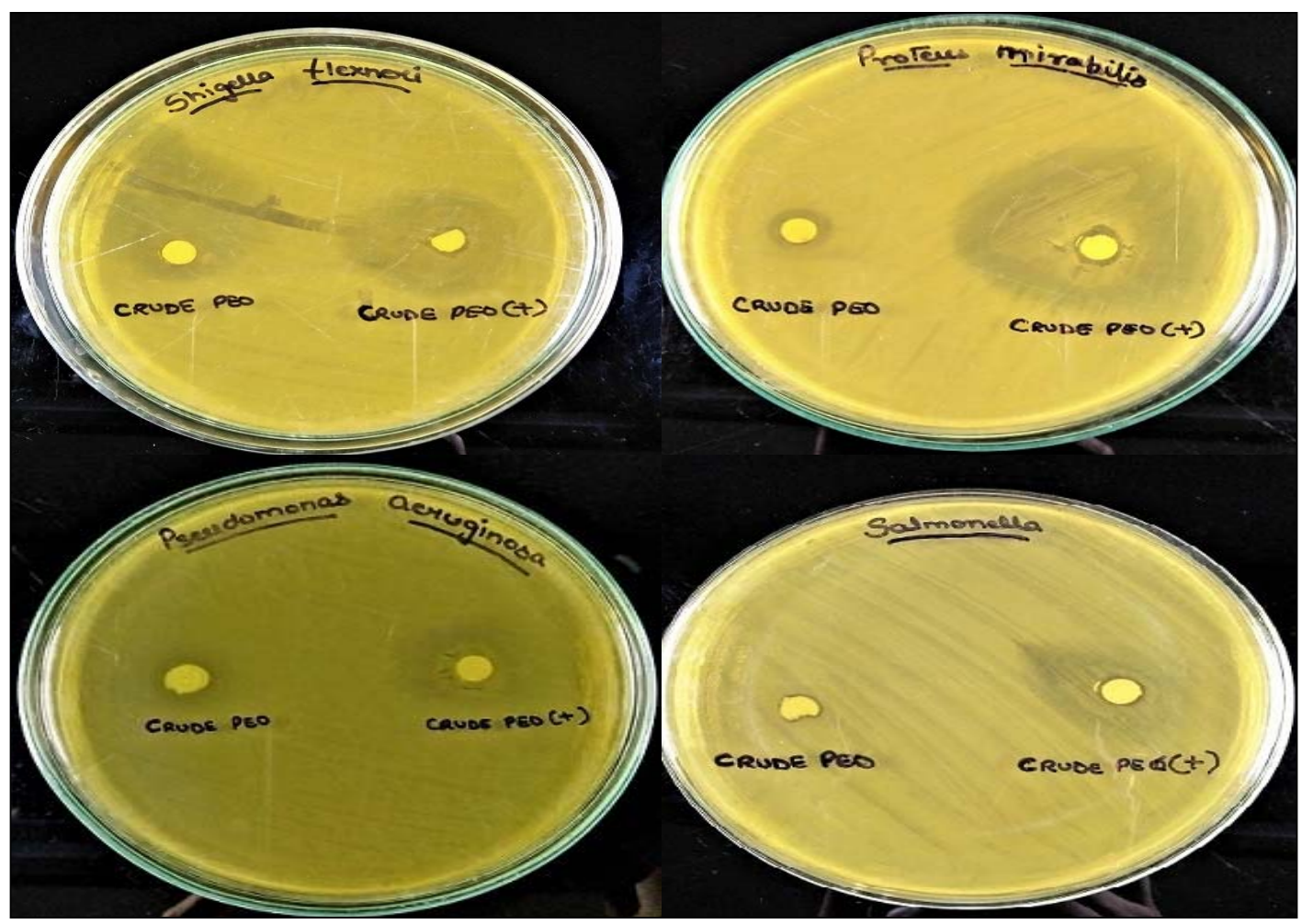

Fig. 1: Zone of inhibition (mm) of Peppermint essential oil (PEO) against food spoilage microorganisms on Mueller Hinton agar 
organisms was observed in the range of 5000-20000 ppm. A significant correlation was also observed between MIC and ZOI values. It was observed that the peppermint essential oil was showing effective results against both Gram-positive as well as Gram-negative microorganisms.

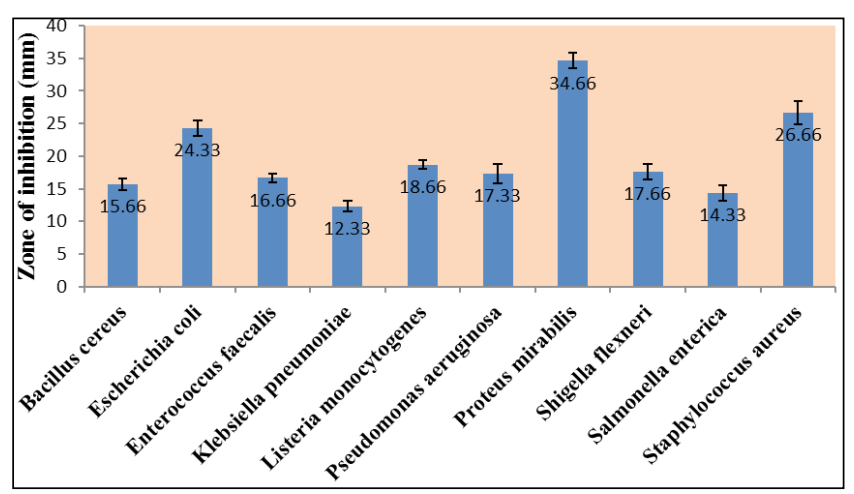

Fig. 2: Zone of inhibition assay $(\mathrm{mm})$ of Peppermint Essential Oil against ten food spoilage microorganisms (Mean \pm S.E.), $n=3$

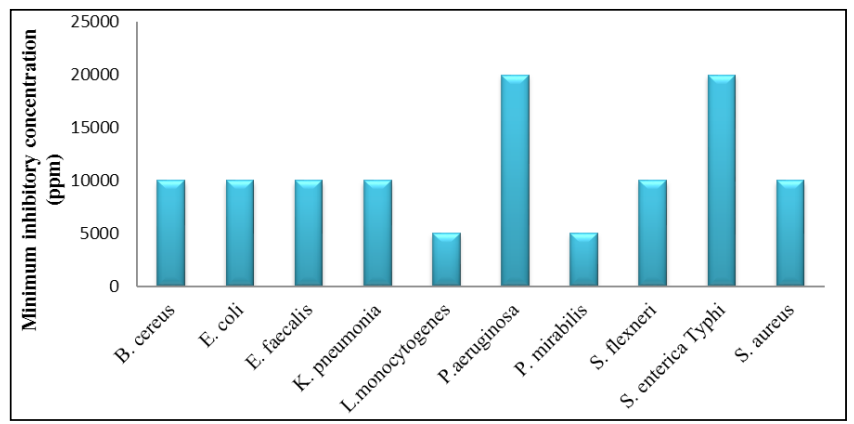

Fig. 3: Minimum Inhibitory Concentration (ppm) of Peppermint Essential Oil against ten food spoilage microorganisms (Mean values), $n=3$

However, the Gram-negative organisms were found slightly more susceptible than Gram-positive organisms. Rusenova et al. (2009) reported in-vitro antimicrobial activity of twelve selected essential oils including peppermint essential oil against Gram-positive and Gramnegative microorganisms and found that the peppermint essential oil was effective against all the tested organisms. Mimica- Dukic et al. (2003) conducted a study on the antibacterial activity of essential oils of three Mentha species against a range of five Gram-positive strains and eight Gram-negative strains of bacteria. The results revealed that generally essential oils were more effective against Gram-positive bacteria than Gram-negative strains, but the test strains of multiresistant Gram-negative bacteria also exhibited a remarkable susceptibility to the examined essential oils. In the present study, though a strong positive correlation was observed between zone inhibition diameters and MIC values, however, a little discrepancy of obtained values as compared with previously conducted studies might be due to variation in origin and type of peppermint plant, method of extraction employed, final composition of peppermint essential oil, methodology adopted for evaluation of ZOI and MIC etc. (Kanth et al., 2018).

\section{Antioxidant efficacy of peppermint essential oil}

Results for antioxidant activity viz. DPPH and ABTS results for $\mathrm{PEO}$ are tabulated in Table 1 respectively. DPPH radical-scavenging action is due to their hydrogendonating ability which is directly proportional to the total number of hydroxyl groups.

Table 1: DPPH and ABTS Radical Scavenging potential of Peppermint essential oil (Mean \pm S.E.), $n=3$

\begin{tabular}{llll}
\hline $\begin{array}{l}\text { SI. } \\
\text { No. }\end{array}$ & $\begin{array}{l}\text { Tested } \\
\text { Concentrations } \\
\text { (ppm) }\end{array}$ & $\begin{array}{l}\text { DPPH Radical } \\
\text { Scavenging } \\
\text { Activity (\%) }\end{array}$ & $\begin{array}{l}\text { ABTS Radical } \\
\text { Scavenging } \\
\text { Activity (\%) }\end{array}$ \\
\hline 1 & 5000 & $35.16 \pm 1.63$ & $17.24 \pm 1.73$ \\
2 & 10000 & $41.76 \pm 1.19$ & $30.96 \pm 1.45$ \\
3 & 15000 & $48.42 \pm 1.65$ & $36.33 \pm 1.55$ \\
4 & 20000 & $55.87 \pm 1.36$ & $46.21 \pm 1.04$ \\
5 & 25000 & $60.70 \pm 1.08$ & $49.07 \pm 1.47$ \\
\hline
\end{tabular}

Therefore, more the number of hydroxyl groups, the greater is the hydrogen-donating ability and higher is the DPPH radical-scavenging activity (Blois, 1958). DPPH radical scavenging activity of oil was determined by using method of Kumar et al. (2017) with slight modification. A solution of DPPH $(0.15 \mathrm{mmol} / \mathrm{L})$ in methanol was used along with five different concentrations of oil $(5000,10000$, $15000,20000,25000 \mathrm{ppm}$ ) and it was observed that there was an increasing trend of radical scavenging activity with increase in the concentration of peppermint essential oil. The different concentrations of essential oil to be tested were selected on the basis of MIC values as depicted in Fig. 3. ABTS decolourization is an excellent tool for the evaluation of antioxidant activity of hydrogen donating antioxidants. It was observed that there was an increasing 


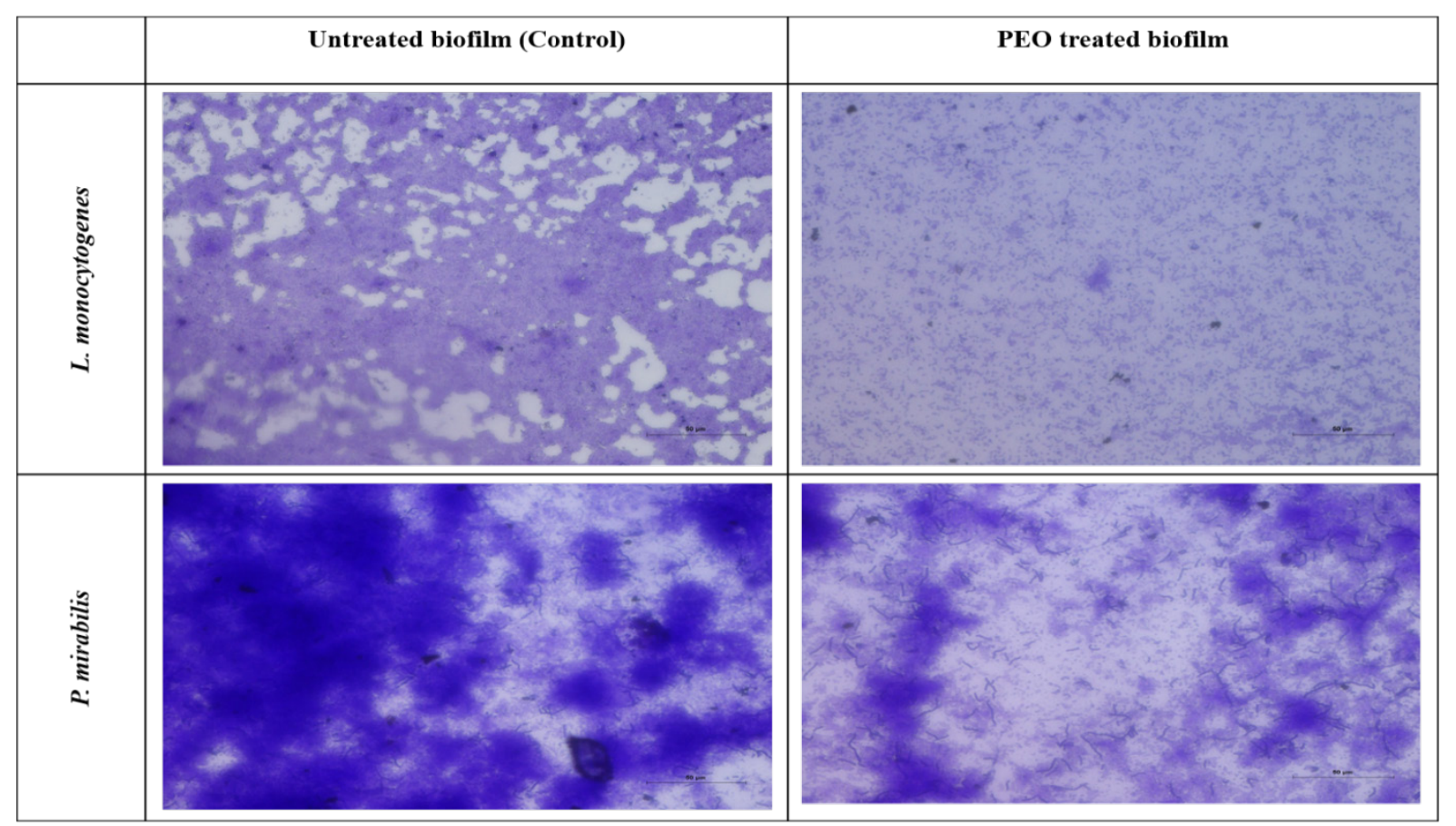

Fig. 4: Light microscopic images of biofilms of L. monocytogenes and P.mirabilis grown in the absence and presence of peppermint essential oil (PEO)

trend of radical scavenging activity with increase in the concentration of peppermint essential oil. The presence of active principle i.e. menthol might be responsible for the higher radical scavenging activity of peppermint oil. The findings indicate that essential oil under investigation has potential antioxidant and free radical scavenging activity. Along with proven health benefits, these attributes can be helpful in controlling oxidative rancidity in high fat food products such as meat, thereby extending its storage stability.

\section{Antibiofilm activity of peppermint essential oil}

In our study, we had explored the effect of peppermint essential oil on formation of biofilms by pathogens. The obtained results demonstrated that peppermint oil inhibited biofilm formation by $L$. monocytogenes and $P$. mirabilis strains with 45.80 and $73.01 \%$, respectively (Fig. 4). In addition, the obtained results showed a correlation with anti-microbial activity. Light microscopic analysis revealed that the control slides showed a well-developed biofilm growth of test bacterial pathogens, whereas, pathogens treated with the peppermint oil (5000 ppm) showed poor and loose biofilm architecture that established its promising ability to destroy the biofilm structures. Similarly, Bazargani et al. (2016) reported that peppermint essential oil showed highest activity in the inhibition of biofilm growth against S.aureus (Gram positive) and E.coli (Gram negative) bacteria.

\section{CONCLUSION}

In vitro antimicrobial, antioxidant and antibiofilm study of peppermint essential oil reveals that it has potential antimicrobial ability and good radical scavenging activity against common food spoilage and therefore possessing broad spectrum activity against both Gram positive and Gram negative organisms. In addition, it also possess capability to inhibit formation of biofilm by pathogenic bacteria. Thus, peppermint essential oil can be used as a substitute of synthetic additives (antimicrobials and antioxidants) in order to control the food spoiling pathogens. Moreover, it can be tried as potential natural preservatives in high fat foodstuffs, especially the meat products as an aromatic and flavoring component without affecting the organoleptic quality and can also prevent 
the oxidative rancidity during storage at refrigeration temperature which ultimately enhances shelf life of the product.

\section{ACKNOWLEDGEMENTS}

The authors are grateful to Ministry of Food Processing Industries (MoFPI) and SERB, DST Government of India for financial support in the form of project entitled "Encapsulation of Natural Bioactive Compounds and Micronutrients for the Enhancement of Nutritive, Preservative and Processing Functionality of Meat Products" to carry out this work (SERB/ MOFPI/0025/2014).

\section{CONFLICT OF INTEREST}

On behalf of all authors, the corresponding author states that there is no conflict of interest.

\section{REFERENCES}

Adorjan, B. and Buchbauer, G. 2010. Biological properties of essential oils: an updated review. Flavour Frag. J., 25(6): 407-426.

Bauer, A.W., Kirby, W.M.M., Sherris, J.C. and Turck, M. 1966. Antibiotic susceptibility testing by a standardized single disk method. Am J. Clin. Pathol., 45: 493-496.

Bazargani, M.M. and Rohloff, J. 2016. Antibiofilm activity of essential oils and plant extracts against Staphylococcus aureus and Escherichia coli biofilms. Food Control., 61: 156-164.

Blois, M.S. 1958. Antioxidant determinations by the use of a stable free radical. Nature, 181(4617): 1199.

Burt, S. 2004. Essential oils: their antibacterial properties and potential applications in foods-a review. Int. J. Food Microbiol., 94(3): 223-253.

De Gonzalez, M.N., Hafley, B.S., Boleman, R.M., Miller, R.K., Rhee, K.S. and Keeton, J.T. 2008. Antioxidant properties of plum concentrates and powder in precooked roast beef to reduce lipid oxidation. Meat Sci., 80(4): 997-1004.

Galvao, N.N., Chiarini, E., Destro, M.T., de Aguiar Ferreira, M. and Nero, L.A. 2012. PFGE characterisation and adhesion ability of Listeria monocytogenes isolates obtained from bovine carcasses and beef processing facilities. Meat Sci., 92(4):635-643.

Jayathilakan, K., Sharma, G.K., Radhakrishna, K. and Bawa, A.S. 2007. Antioxidant potential of synthetic and natural antioxidants and its effect on warmed-over-flavour in different species of meat. Food Chem., 105(3): 908-916.

Kamatou, G.P., Vermaak, I., Viljoen, A.M. and Lawrence, B.M. 2013. Menthol: a simple monoterpene with remarkable biological properties. Phytochem., 96: 15-25.

Kanatt, S.R., Chander, R. and Sharma, A. 2008. Chitosan and mint mixture: A new preservative for meat and meat products. Food Chem., 107(2): 845-852.

Kanth, M.K., Mehta, N., Chatli, M.K., Malav, O.P., Kumar, P., Wagh, R.V. and Panwar, H. 2018. In-vitro assessment of antimicrobial, antibiofilm and antioxidant potential of essential oil from Rosemary (Rosmarinus officinalis L.). $J$. Anim. Res., 8(6): 1-10.

Kumar, D., Mehta, N., Chatli, M. K., Kaur, G., Malav, O.P. and Kumar, P. 2017. In-vitro assessment of antimicrobial and antioxidant potential of essential oils from Lemongrass (Cymbopogon citratus), Cinnamon (Cinnamomum verum) and Clove (Syzygium aromaticum). J. Anim. Res., 7(6): 10991105.

Kumar, P., Mishra, S., Malik, A. and Satya, S. 2011. Insecticidal properties of Mentha species: a review. Ind. Crops Prod., 34(1): 802-817.

Lund, M.N., Heinonen, M., Baron, C.P. and Estevez, M. 2011. Protein oxidation in muscle foods: A review. Mol. Nutr. Food Res., 55(1): 83-95.

Maffei, M. and Mucciarelli, M. 2003. Essential oil yield in peppermint/soybean strip intercropping. Field Crops Res., 84(3): 229-240.

Mehta, N., Ahlawat, S.S., Sharma, D.P., Yadav, S. and Arora, D. 2013. Development and quality evaluation of chicken patties incorporated with psyllium husk. Haryana Vet., 52(2): 6-11.

Mimica-Dukic, N., Bozin, B., Sokovic, M., Mihajlovic, B. and Matavulj, M. 2003. Antimicrobial and antioxidant activities of three Mentha species essential oils. Planta Med., 69(5): 413-419.

Musthafa, K.S., Ravi, A.V., Annapoorani, A., Packiavathy, I.S.V. and Pandian, S.K. 2010. Evaluation of anti-quorum-sensing activity of edible plants and fruits through inhibition of the $\mathrm{N}$-acyl-homoserine lactone system in Chromobacterium violaceum and Pseudomonas aeruginosa. Chemotherapy. 56(4): 333-339.

Naveena, B.M., Sen, A.R., Vaithiyanathan, S., Babji, Y. and Kondaiah, N. 2008. Comparative efficacy of pomegranate juice, pomegranate rind powder extract and BHT as antioxidants in cooked chicken patties. Meat Sci., 80(4): 1304-1308.

Raghavan, S. and Richards, M.P. 2007. Comparison of solvent and microwave extracts of cranberry press cake on the inhibition of lipid oxidation in mechanically separated turkey. Food Chem., 102(3): 818-826. 
Raut, J.S. and Karuppayil, S.M. 2014. A status review on the medicinal properties of essential oils. Ind. Crops Prod., 62: 250-264.

Re, R., Pellegrini, N., Proteggente, A., Pannala, A., Yang, M. and Rice-Evans, C. 1999. Antioxidant activity applying an improved ABTS radical cation decolorization assay. Free Radic. Biol.Med., 26(9-10): 1231-1237.

Rusenova, N. and Parvanov, P. 2009. Antimicrobial activities of twelve essential oils against microorganisms of veterinary importance. Trakia J. Sci., 7(1): 13-15.

Saeed, S., Naim, A.S., Naim, M.A. and Tariq, P. 2006. In vitro antibacterial activity of peppermint. Pak. J. Bot., 38(3): 869.

Sallam, K.I. and Samejima, K. 2004. Microbiological and chemical quality of ground beef treated with sodium lactate and sodium chloride during refrigerated storage. LWT-Food Sci. Technol., 37(8): 865-871.

Sebranek, J.G. and Bacus, J.N. 2007. Cured meat products without direct addition of nitrate or nitrite: what are the issues. Meat Sci., 77(1): 136-147.

Shah, P.P. and Mello, P.M.D. 2004. A review of medicinal uses and pharmacological effects of Mentha piperita. Nat Prod Rad., 3(4): 214-221.
Shimada, K., Fujikawa, K., Yahara, K. and Nakamura, T. 1992. Antioxidative properties of xanthan on the autoxidation of soybean oil in cyclodextrin emulsion. J. Agr. Food Chem., 40(6): 945-948.

Shree, P., Mehta, N., Chatli, M.K., Wagh, R.V. and Panwar, H. 2019. In-vitro evaluation of antimicrobial and antioxidant Efficacy of thyme (Thymus vulgaris L.) essential oil. J. Anim. Res., 9(3): 443-449.

Snedecor, G. W. and Cochran, W. G. 1994. Statistical Methods, 8th Edn. Ames: Iowa State Univ. Press Iowa.

Verma, A.K., Chatli, M.K., Mehta, N. and Kumar, P., 2018. Assessment of physico-chemical, antioxidant and antimicrobial activity of porcine blood protein hydrolysate in pork emulsion stored under aerobic packaging condition at $4 \pm 1^{\circ} \mathrm{C}$. LWT-Food Sci. Technol., 88: 71-79.

Yen, G.C. and Duh, P.D. 1994. Scavenging effect of methanolic extracts of peanut hulls on free-radical and active-oxygen species. J. Agr. Food Chem., 42(3): 629-632. 